\title{
Screening for fetal chromosome aberrations in early pregnancy
}

\author{
M. A. FERGUSON-SMITH AND M. E. FERGUSON-SMITH
}

From the University Department of Medical Genetics, Royal Hospital for Sick Children, Yorkhill, Glasgow

The development of techniques for the prenatal diagnosis of severe genetic disorders in the fetus must be regarded as one of the most important advances in clinical practice in the past ten years (for review see Milunsky, 1973; Harris, 1974; FergusonSmith, 1974). When applied to pregnancies recognized to be at risk of chromosome aberrations, open neural tube defects and a variety of other genetic disorders, prenatal diagnosis and selective termination of affected pregnancies can lead to a substantial reduction in the birth incidence of these disorders and allow families at risk the opportunity of having normal children. It appears that the procedure, available only in the larger centres in Britain, is acceptable to the majority of patients and practitioners. The demand for prenatal diagnosis is increasing rapidly at present (fig 1 ) and there is considerable pressure to make it more generally available. It therefore seems an appropriate time to review past experience, and consider in which situations prenatal diagnosis has been shown to be of most value so that sensible decisions can be made about the type of service to be provided in the future.

This paper reviews seven years' experience in providing prenatal screening for fetal chromosome aberrations in the west of Scotland. In addition to providing useful information on the indications for prenatal diagnosis, on the frequency of fetal chromosome aberrations in certain groups at risk, on the benefits to individual families and to the community, our analysis has implications for the future planning of prenatal diagnostic services.

\section{Materials and Methods}

Patients are referred from a variety of sources, including general practice, and from obstetric, paediatric and other specialist clinics, and many are seen first through the genetic advisory service. Before being accepted for amniocentesis the pregnant woman and her husband are given genetic counselling and advised about the risks of amniocentesis, the limitations of the diagnostic technique and the possibility that the amniocentesis might have to be

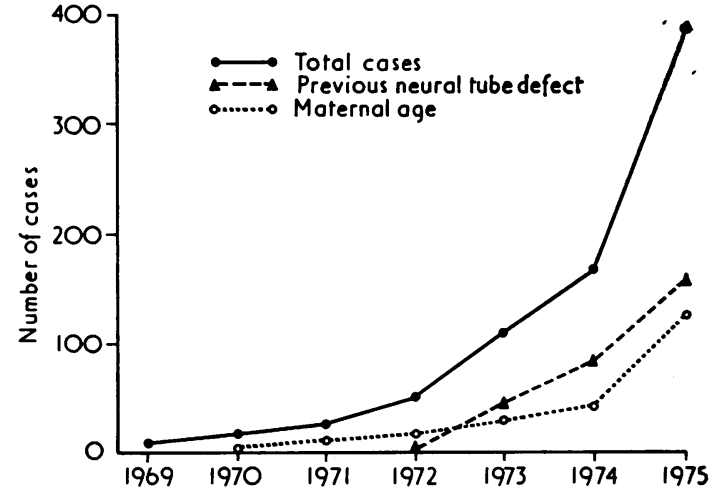

Fig 1 The increasing demand for prenatal diagnosis; annual totals in the west of Scotland for the period 1969 to 1975.

repeated. Many of the patients are seen at the genetics clinic before becoming pregnant to assess the risk to future pregnancies and all but a few have indicated clearly that they would agree to termination in the event that a fetal abnormality is detected. In all cases, it is made clear that the prospective parents are free to decide whether or not to accept the option of prenatal diagnosis.

A sterile amniotic fluid sample, usually $15-20 \mathrm{ml}$ but sometimes much less, is obtained by transabdominal amniocentesis at about 16 weeks' gestation (range 14-24 weeks). The preferred procedure is that the patient attends the Ultrasonography Department as an outpatient and amniocentesis is performed under aseptic conditions using a local anaesthetic and under the direct guidance of ultrasound. The fetal heart activity is checked before and after amniocentesis and all $\mathrm{Rh}$-negative mothers receive $100 \mu \mathrm{g}$ of anti-D gammaglobulin after amniocentesis to protect them from the possible hazard of isoimmunization.

The majority of amniotic fluid samples are received in the laboratory on the day of amniocentesis. One to $2 \mathrm{ml}$ of the specimen is taken for $\mathrm{X}$ - 
chromatin analysis. This sample is diluted in $100 \mathrm{ml}$ of normal saline and filtered through an SM Millipore filter, washed with normal saline and the amniotic fluid cells fixed in situ on the filter using $95 \%$ ethanol. The complete filter is stained in $1 \%$ cresyl echt violet and mounted, face up, in DePeX on a standard microscope slide. Only well fixed amniotic cell nuclei are scored for X-chromatin. A further 1-2 ml of amniotic fluid is added to an equal volume of freshly prepared alcohol:acetic acid fixative $(3: 1)$ and is centrifuged after $10-15$ minutes. Of the cell deposit, $0.75 \mathrm{ml}$ resuspended in fixative is used to make air-dried slides in a cytocentrifuge (Shandon). These preparations are stained in $0.5 \%$ aqueous quinacrine dihydrochloride and examined directly under the ultraviolet microscope for the frequency of fluorescent Y-chromatin in amniotic cell nuclei.

The remainder of the amniotic fluid sample is used to prepare amniotic cell cultures by a modification of an in-situ method, previously described (Ferguson-Smith et al, 1971). The sample is centrifuged for 20 minutes at $250 \mathrm{~g}$, and the cell deposit (resuspended in $1 \mathrm{ml}$ of amniotic fluid) is divided equally between four $30 \mathrm{~mm}$ plastic petri dishes. The cells are covered by a sterile coverslip, and $2 \mathrm{ml}$ of culture medium (Ham's F.10 $+30 \%$ fetal calf serum) is added to each dish and the dishes are incubated at $37^{\circ} \mathrm{C}$ in $5 \% \mathrm{CO}_{2}$. The cultures are continued, replacing the medium every three days, until an active monolayer is apparent (average seven to 10 days). The coverslip is removed into a new dish with fresh medium and inspected again to ensure that the monolayer is on the coverslip and not in the dish. Eight $\mu \mathrm{g}$ of colcemid is added $24 \mathrm{hr}$ later and allowed to act at $37^{\circ} \mathrm{C}$ for five to six hours. The medium is replaced with $0.075 \% \mathrm{KCl}$ at $37^{\circ} \mathrm{C}$ for
40 to 50 minutes, and the cells are fixed by adding $1 \mathrm{ml}$ of fresh 3:1 absolute alcohol:glacial acetic acid to the $\mathbf{K C l}$ in the dish. After 30 seconds the fluid in the dish is replaced by fresh fixative for $\mathbf{1 0}$ minutes. The coverslip is removed, dried quickly in air and stained. Although it is important to make the diagnosis on the primary cultures, the cells which remain attached to the petri dish after removal of the coverslip can be redistributed by trypsinization and used to make fresh coverslip preparations or airdried drop preparations of cells fixed in suspension. Routinely, the first coverslip to be harvested is stained in $2 \%$ aceto-orcein and if adequate counts are obtained, subsequent coverslips are stained by the ASG method (Sumner et al, 1972), the trypsinLeishman method (Seabright, 1973) or by the Q banding methods, depending on what is considered appropriate to the case. The fetal karyotype is determined separately from at least two primary cultures in each case. In each culture, the mitoses of each clone are analysed separately so that aberrations arising in vitro can be quickly identified. Particular attention is paid to clones of fibroblast type which may be maternal in origin.

In all terminations, the prenatal diagnosis is checked by chromosome analysis of cell cultures derived from the fetal tissue.

\section{Results}

During the period $1969-75,353$ pregnant women at risk of fetal chromosome aberrations were tested in early pregnancy. This represents about $46 \%$ of all referrals for prenatal diagnosis. A further 363 cases were tested to exclude a coincidental fetal chromosomal abnormality although the primary indication was non-chromosomal.

\begin{tabular}{|c|c|c|c|c|c|c|}
\hline \multirow[t]{2}{*}{ Indication } & \multirow{2}{*}{$\begin{array}{l}\text { Number of } \\
\text { Pregnancies }\end{array}$} & \multicolumn{2}{|c|}{ Number of Abnormalities } & \multirow{2}{*}{$\begin{array}{l}\text { Percentage } \\
\text { Abnormalities }\end{array}$} & \multirow{2}{*}{$\begin{array}{l}\text { Number } \\
\text { Terminated }\end{array}$} & \multirow{2}{*}{$\begin{array}{l}\text { Number } \\
\text { Miscarried }\end{array}$} \\
\hline & & Detected & Missed & & & \\
\hline \multicolumn{7}{|l|}{ Primary } \\
\hline Previous spina bifida, etc & 296 & 13 & $5^{1}$ & $6 \cdot 2$ & 11 & 6 \\
\hline High maternal serum AFP & 21 & 9 & 0 & $42 \cdot 9$ & 9 & $\mathbf{0}$ \\
\hline Previous trisomic mongolism & 104 & 1 & 0 & $1 \cdot 0$ & 1 & 2 \\
\hline Maternal age, 35-39 years & 72 & 1 & 0 & $1 \cdot 4$ & $i$ & $\overline{1}$ \\
\hline Maternal age, 40 years and over & 121 & 7 & 1 & 6.6 & 7 & 2 \\
\hline Chromosomal translocation/mosaicism & 20 & 1 & 0 & $5 \cdot 0$ & 1 & $\mathbf{0}$ \\
\hline Other chromosomal indications & 37 & 1 & $\mathbf{0}$ & $2 \cdot 7$ & 1 & $\mathbf{0}$ \\
\hline X-linked disease & 16 & 4 & $\mathbf{0}$ & $23 \cdot 5$ & 3 & $\mathbf{0}$ \\
\hline Autosomal recessive disease & 16 & 3 & 0 & $20 \cdot 0$ & 2 & $\mathbf{0}$ \\
\hline Others & 54 & 4 & 0 & $7 \cdot 3$ & 4 & 1 \\
\hline Total & 761 & & & & & \\
\hline \multicolumn{7}{|l|}{ Secondary } \\
\hline Exclude spina bifida & (393) & 1 & $1^{1}$ & 0.5 & 1 & - \\
\hline Exclude chromosome abnormality & (363) & 3 & 0 & 0.8 & $2(+1)$ & 一 \\
\hline Total & 761 & 48 & 7 & $7 \cdot 2$ & 43 & $12(1 \cdot 6 \%)$ \\
\hline
\end{tabular}

Table I Results of prenatal diagnosis in the west of Scotland 1969-1975

'Closed neural tube defects. 
Table I shows that the most frequent indication for fetal chromosome analysis in our series is increased maternal age. In 121 mothers 40 years of age or over, an unbalanced chromosome aberration was detected in seven pregnancies and missed in one pregnancy. In another pregnancy a balanced 4/6 translocation (fig 2C) was unexpectedly discovered; the father was later shown to carry the same translocation. Without including this case, the abnormality rate in pregnancies in this maternal age category is $6.6 \%$ and the eight individual aberrations comprised seven cases of trisomy-21 Down's syndrome (fig 2A), and one case of XXY Klinefelter's syndrome (fig 2B). Among the 72 women who were tested because they were between the ages of 35 and 39 years, only one pregnancy $(1.4 \%)$ was abnormal and this proved to be affected with XXY Klinefelter's syndrome.

One hundred and four mothers who had a child with trisomic Down's syndrome requested amnio- centesis because of anxiety about the possiblity of recurrence and only one pregnancy was found to have trisomy 21 . This case was exceptional in that the couple had two previous children with trisomy 21 and one of the parents could well have been a chromosomal mosaic, although lymphocyte and fibroblast cultures showed no evidence of this.

Parental chromosomal translocations and mosaicism have been comparatively rare indications for prenatal diagnosis. Theoretically one expects approximately half of the conceptions of a parent with a balanced translocation to be chromosomally abnormal. However, an unbalanced karyotype was found in only one of the 18 patients in this group. In this case the mother had a balanced 9/14 translocation with breakpoints at 9q22 and 14q11 (fig 3A). The fetus had a duplication of the short arm of chromosome 9 (fig 3B) and would have been expected to develop the $9 p$ trisomy syndrome. Of the remaining 17 cases in this group, eight fetuses had

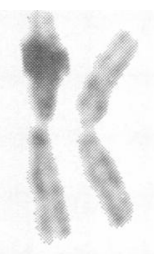

I

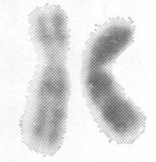

7

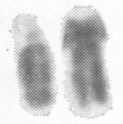

13

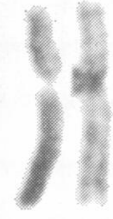

2

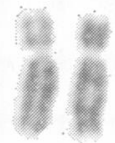

8

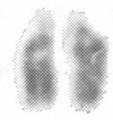

14

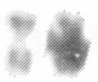

20

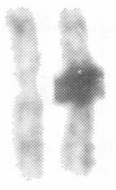

3

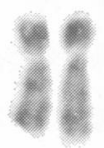

9

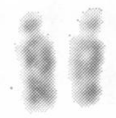

15

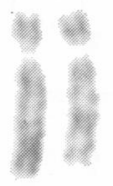

4

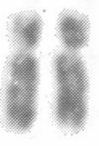

10

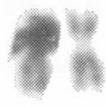

16

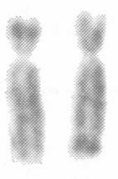

5

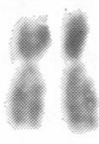

II

12

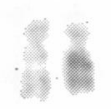

17

18

Fig 2A

Fig 2 Fetal karyotypes in a pregnancy tested because of maternal age affected with:

$\mathbf{X}$

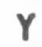

A Trisomic Down's syndrome 


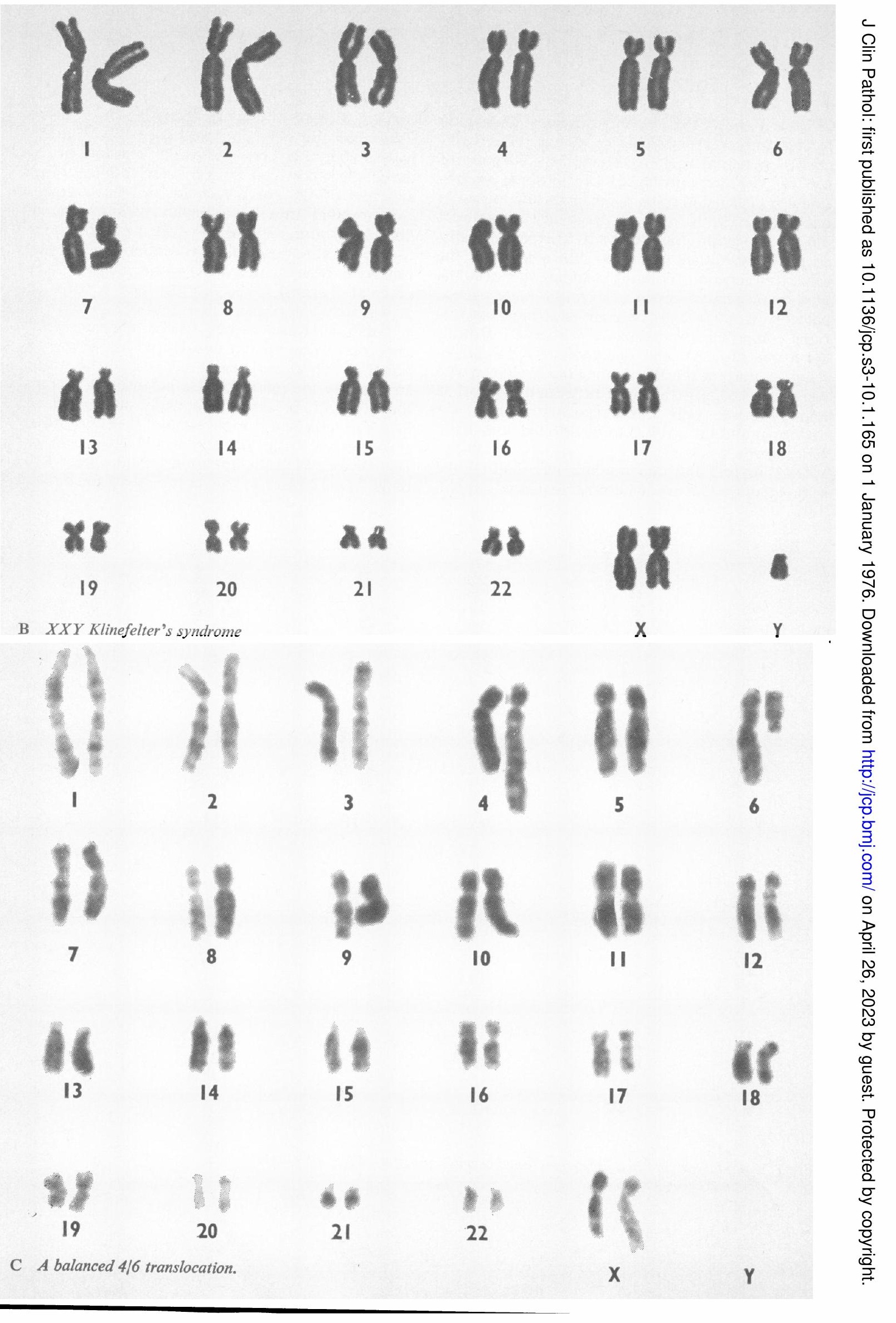




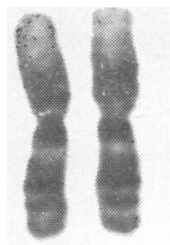

I

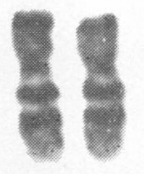

7

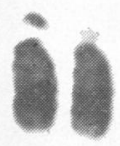

13

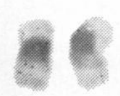

19

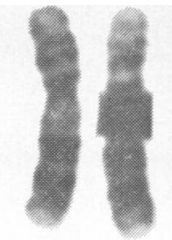

2

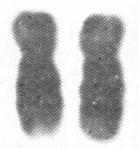

8

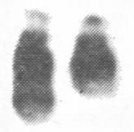

14

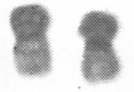

20

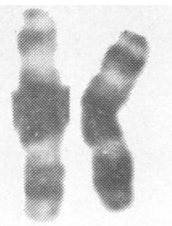

3

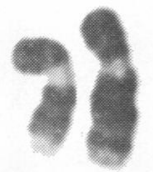

9

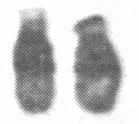

15

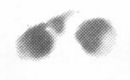

21

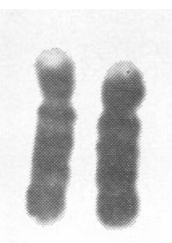

4

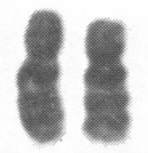

10

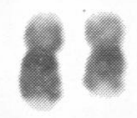

16

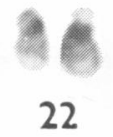

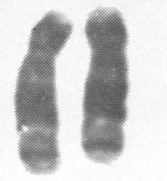

5

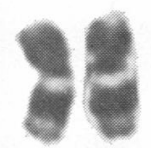

| |

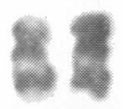

17
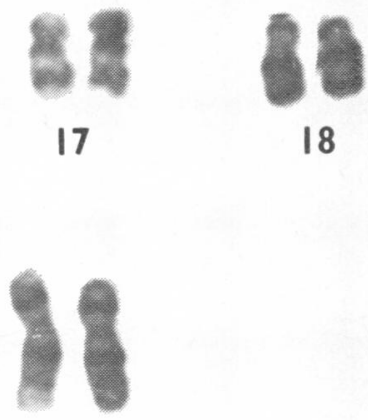

X

Fig 3A

Fig 3 An unbalanced translocation found in the pregnancy of a mother heterozygous for a $9 / 14$ reciprocal translocation. A Maternal karyotype, 46, XX,t(9;14) $(q 22 ; q 11)$.

balanced translocations like the carrier parent and nine had entirely normal karyotypes. Eight of the translocation parents were heterozygous for a centric fusion translocation (five 13/14 and three $14 / 21$ translocations), nine had a balanced reciprocal translocation and one had a non-reciprocal insertional translocation. We also include in this category in table I two cases of maternal chromosomal mosaicism, one for normal and trisomy 21 cells, the other for normal and trisomy 18 cells; both fetuses had normal female karyotypes.

Among a miscellaneous group of cases referred for fetal chromosome analysis ('other chromosomal' in table $\mathrm{I}), 22$ had an affected parental sibling or other more distant relative affected with Down's syndrome, six had a previous child affected with trisomy 18, two had a previous child affected with trisomy 13 and four had children with other chromosome aberrations. In only one of this group was a chromosome aberration found and this was clearly fortuitous. The husband's nephew had 21/21 trans- location Down's syndrome and the obstetrician performed amniocentesis under the mistaken impression that the aberration was familial. An X/19 translocation, with breakpoints at Xq24 and 19q13, was found in the mother; however, only one translocation product could be identified in the fetus and the pregnancy was terminated.

In pregnancies of women who are known carriers of $\mathrm{X}$-linked recessive disease, cytogenetic studies are an important part of prenatal diagnosis. In a few disorders such as the Lesch-Nyhan syndrome, Hunter's syndrome and Fabry's disease, a specific biochemical test can be applied to amniotic cell cultures. Four pregnancies at risk of Hunter's syndrome (mucopolysaccharidosis II) were tested in this series. Sex chromatin and chromosome analysis showed that two of the fetuses were male and two were female. However, $\mathbf{S}^{\mathbf{3 5}}$ sulphate incorporation studies and other tests on amniotic cell cultures were judged to be normal and all pregnancies continued successfully to term with the birth of normal 


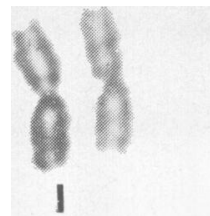

1
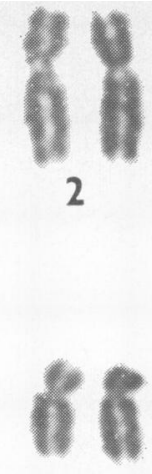

8

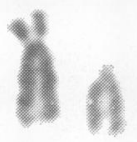

14

13
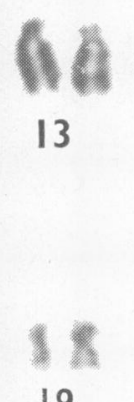

19

Fig 3B

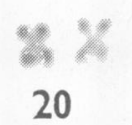

B Fetal karyotype, 46, XY, -14, $+\operatorname{der}(9), t(9 ; 14)(q 22 ; q 11)$ mat

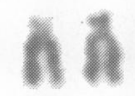

15
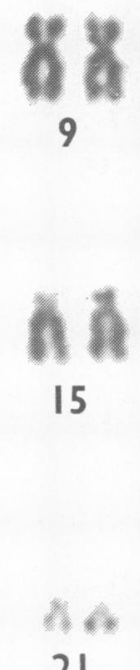

21

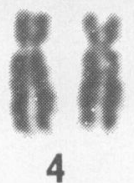

4
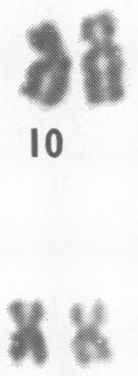

16
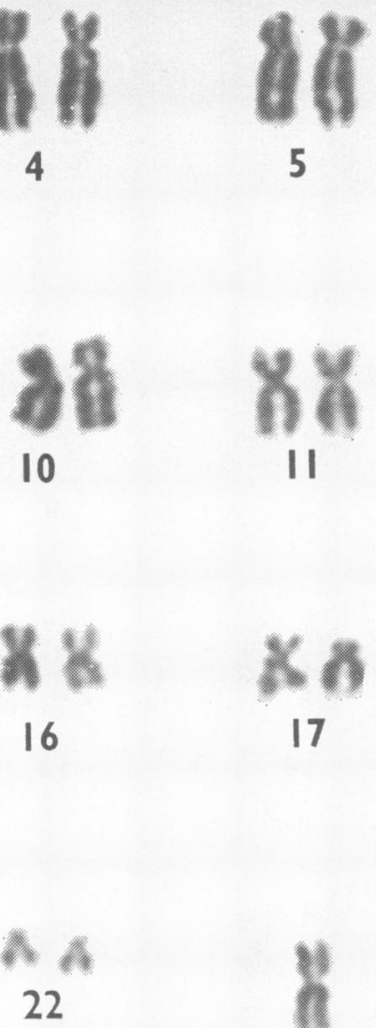

17
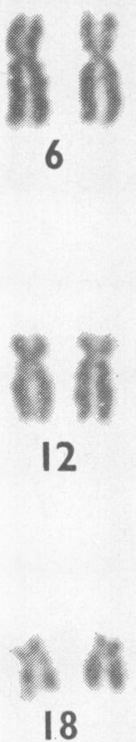
the long arm of chromosome 9.

children of the predicted sex. (It is hoped to present full details of these cases elsewhere.) Twelve pregnancies were at risk for X-linked disorders which could not be specifically diagnosed in utero. Fetal sexing was undertaken in these cases to give the parents the option of selective termination should the fetus be male and consequently have a $50 \%$ chance of being affected. In the five pregnancies at risk of Duchenne muscular dystrophy fetal sex was found to be female in four and so only one pregnancy was terminated. Although both cases at risk of haemophilia proved to have male fetuses, the option of termination was only taken in one; the other pregnancy resulted in a normal unaffected male. In the two cases at risk of chronic granulomatous disease, both were male but only one was terminated; fetal chromosome analysis showed that this fetus was also affected by trisomic Down's syndrome. In the remaining single pregnancies at tisk of $\mathrm{X}$-linked hydrocephalus, spondyloepiphyseal dys- plasia tarda and the Wiskott-Aldrich syndrome, the fetal sex was female and the pregnancies were allowed to continue to term. In all cases $X$ and $Y$ chromatin analysis gave correct predictions of the fetal sex within 48 hours of the amniocentesis, although confirmation by fetal chromosome analysis was usually sought before termination.

In addition to the 353 pregnancies tested because of an increased risk of fetal chromosome abnormality, or for the determination of fetal sex, prenatal chromosome analysis was also undertaken in 363 cases in which the primary indication was not a chromosome aberration; the majority of these were pregnancies at risk of an open neural tube defect. The importance of undertaking fetal chromosome analysis in these cases is shown by the finding that three of the 363 cases had a serious unbalanced chromosome aberration and three were found to have a balanced or non-significant aberration. A pregnancy at risk of a neural tube defect and a preg- 


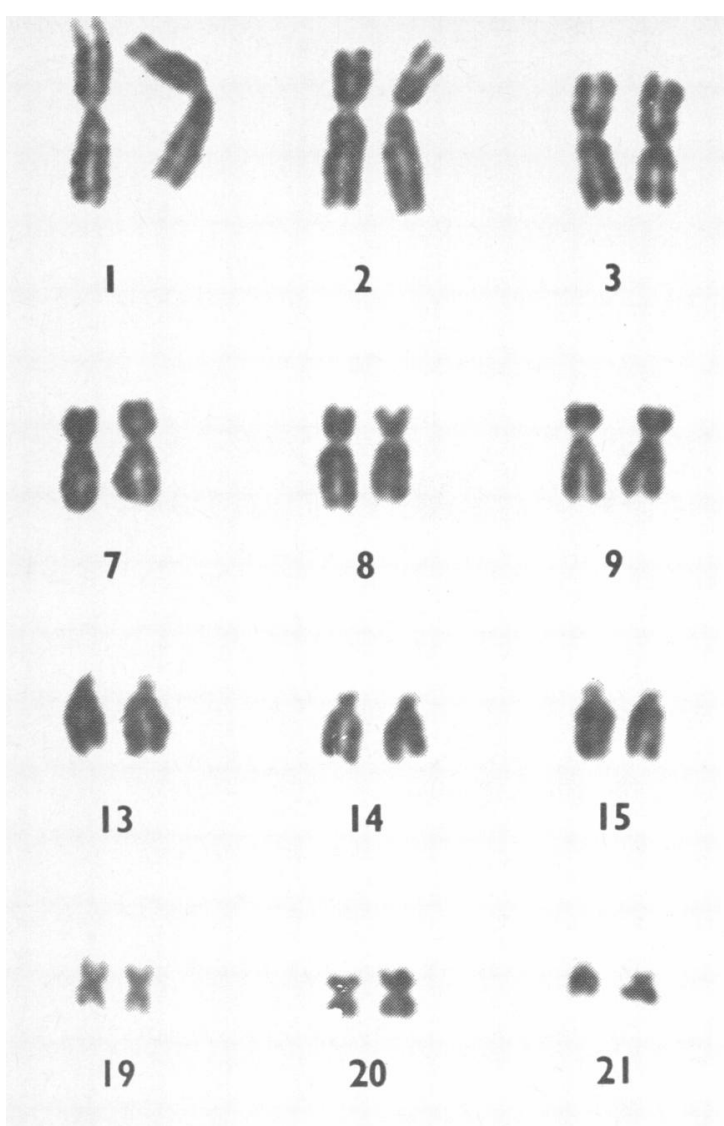

Fig 4A

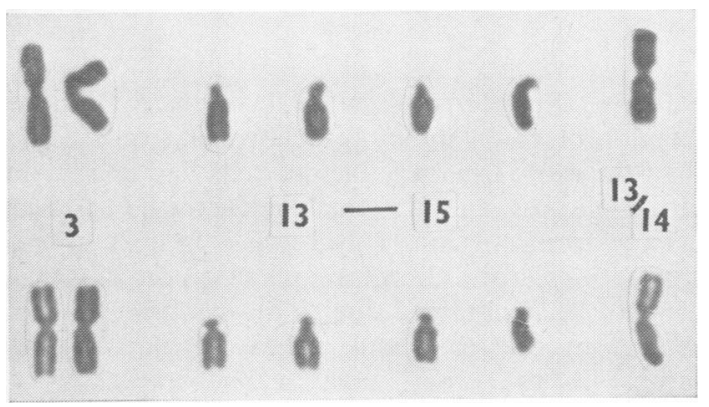

Fig 4B

nancy at risk of chronic granulomatous disease (previously mentiontd), were both found to have trisomy 21 Down's syndrome, and another pregnancy at risk of a neural tube defect had the triple-X syndrome (fig 4A); these three pregnancies were terminated. In another pregnancy at risk of neural tube defect, fetal chromosome analysis showed a balanced 13/14 translocation (fig 4B); neither of the parents were carriers. A small extra metacentric

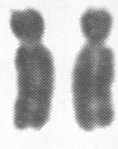

4

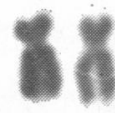

10

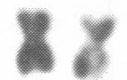

16

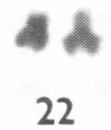

22

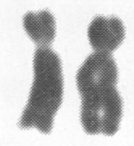

5

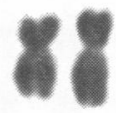

II

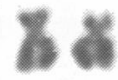

17

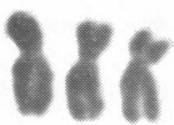

$\mathbf{x}$
Fig 4 A Triple-X karyotype found unexpectedly in a pregnancy at risk for a neural tube defect.

B $A$ de novo balanced 13/14 translocation found unexpectedly in a pregnancy at risk for a neural tube defect.

chromosome satellited at both ends (fig 5) was found in two pregnancies from different families in mothers at risk of Niemann-Pick's disease and a neural tube defect respectively. Following this discovery the parents were tested and both mothers were found to have an extra small metacentric chromosome identical to that found in their fetuses. The mothers were advised that this meant that the extra chromosome was not a significant abnormality and, as the other relevant tests were normal, both pregnancies continued and normal babies are born.

In addition to screening for fetal chromosome aberrations in all samples of amniotic fluid submitted 


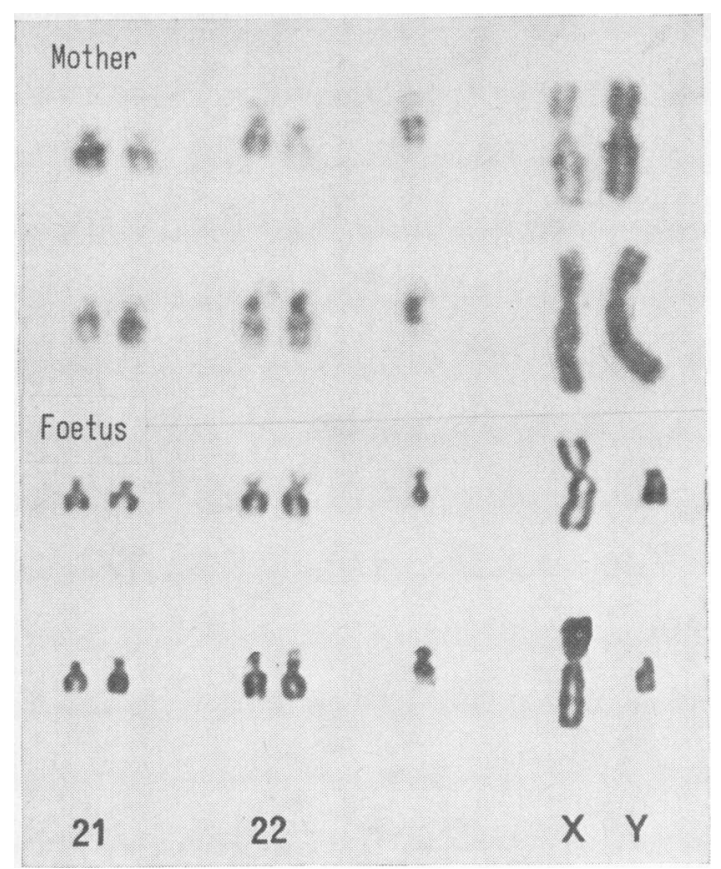

Fig 5A

Fig 5 Partial maternal and fetal karyotypes in two patients whose pregnancies were found unexpectedly to have additional small metacentric chromosomes in all cells. The abnormal chromosomes are satellited at both ends and C-banding reveals that they are largely formed of centric heterochromatin.

irrespective of indication, all samples in our series have had amniotic alphafetoprotein levels estimated. A case of severe fetal spina bifida and another of anencephaly were detected in this way in one patient at risk of Hunter's syndrome and in another tested because of her age.

During the course of fetal chromosome analysis we have observed on several occasions a chromosome aberration which must have arisen in culture (fig 6). In each case, the aberration was identified in only one primary culture and sometimes only in one clone of cells, so that it was clear that the abnormality had arisen as a somatic mutation in culture. No cases of true mosaicism have been identified in our series.

An incorrect fetal chromosomal diagnosis was made in three cases during the early stages of the work and before the hazard of contamination with maternal cells was fully appreciated. Two normal male babies and a female baby with trisomic Down's syndrome were delivered following the prenatal

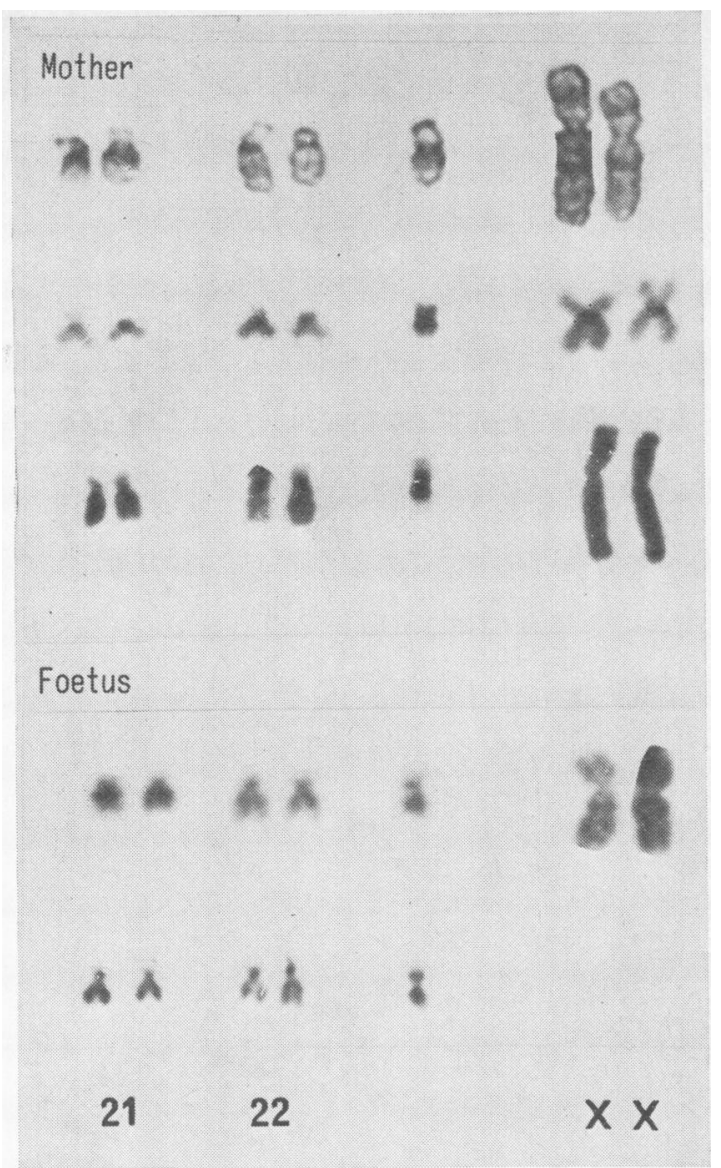

Fig 5B

diagnosis of a normal female karyotype. A review of these three cases shows that fibroblast cells rather than the more usual epithelioid cells had grown out in the cultures, and also that the diagnoses had been derived from only one culture dish. In the two male conceptions, a sex chromatin result on direct smears had not been obtained due to an insufficient $(4 \mathrm{ml})$ sample in one case and a heavily blood-stained sample in the other. Fortunately, only $2.5 \%$ of amniotic cell cultures in our series are exclusively fibroblast in type and these are now reported only if they are male; a second amniocentesis is advised in the others. If this is not possible, it is sometimes helpful to examine the parental karyotypes for chromosomal polymorphisms and determine whether or not the fetal karyotype contains polymorphisms only present in the father. A similar strategy may be used in order to interpret extreme $Y$ chromosome variations in the fetal karyotype. On three occasions, it was reassuring to find that the father had the same 


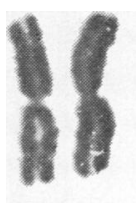

I

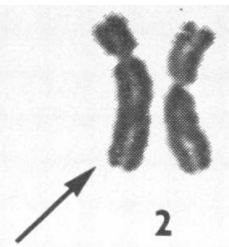

2

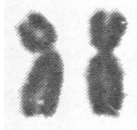

7

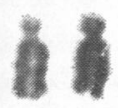

13

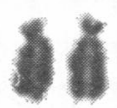

14
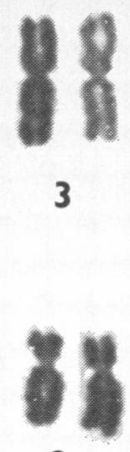

9

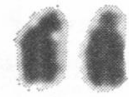

15
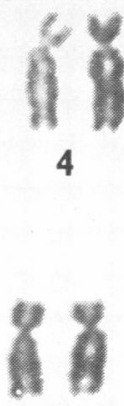

10

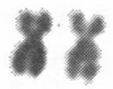

16
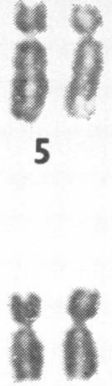

II

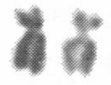

17
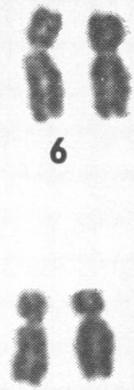

12

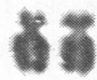

18

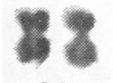

19

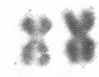

20

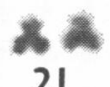

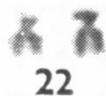

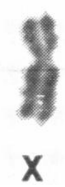

minute non-fluorescent $\mathrm{Y}$ chromosome detected in the fetus.

In the years $1969-74,94 \cdot 3 \%$ of all amniotic fluids tested yielded sufficient cell growth for chromosome analysis. Most of the failures were obtained in samples either sent from a distance (often by air), or inadequate in volume, or contaminated with organisms (12 cases). In 1975, the success rate has increased to $98.5 \%$. In almost all failures, the lack of growth in culture is appreciated in time for a second amniocentesis to be arranged.

In prenatal diagnosis an important consideration is the time taken to reach a chromosomal diagnosis. This period is one of acute anxiety for the patient as she waits to learn whether or not she is to lose her pregnancy. The time taken in culture varies from about five days to 21 days and a further two to three days are required to harvest, stain, analyse and photograph the preparations. On average the report on the fetal karyotype has been available to the patient and her obstetrician on the 18th day after amniocentesis. This means that if termination is required it can usually be carried out by the 20th week of gestation. Induction by prostaglandins is the usual method.

The complications of the procedure to the mother and child appear to be uncommon. The most important is abortion following amniocentesis which in our overall series has occurred in $12(1.6 \%)$ of 761 cases. This rate is little if any higher than the spontaneous abortion rate for all pregnancies after 16 weeks' gestation. Analysis of the first 105 delivered pregnancies and their controls, carefully matched for gestation at time of amniocentesis, similar age ( \pm 3 years), Rhesus group, number of previous livebirths and stillbirths $(0-1,2-4,5$ or more) and number of abortions ( $0-1,2$ or more), show that there have been three abortions in each group and reveal no evidence of serious complications (table II). Nonetheless, amniocentesis is potentially hazardous 


\begin{tabular}{lll}
\hline Complication & \multicolumn{2}{l}{ Amniocentesis } \\
\cline { 2 - 3 } & $\begin{array}{l}\text { Subject } \\
(n=105)\end{array}$ & $\begin{array}{l}\text { Matched } \\
\text { Control } \\
(n=105)\end{array}$ \\
\hline Abortion & 3 & 3 \\
Threatened abortion & 4 & 2 \\
Antepartum haemorrhage & 3 & 4 \\
Isoimmunization & 0 & 0 \\
Stillbirth & 0 & 0 \\
Prematurity & 4 & 1 \\
Fetal malformation & 4 & 3 \\
\hline
\end{tabular}

Table II Frequency of complications in the west of Scotland series

${ }^{1}$ Matched for gestation at time of amniocentesis, similar age $( \pm 3$ years), Rhesus group, number of previous livebirths and stillbirths $(0-1,2-4,5$ or more) and number of abortions $(0-1,2$ or more).

and we believe that it should not be used for trivial indications such as to satisfy curiosity about the fetal sex.

Prenatal screening for fetal chromosome aberration in this series has thus resulted in the selective termination of nine pregnancies affected with trisomic Down's syndrome, two pregnancies affected with XXY Klinefelter's syndrome, two pregnancies affected with a chromosomal translocation and one pregnancy affected with the triple- $X$ syndrome. One fetus affected with trisomic Down's syndrome was missed, and 12 other pregnancies continued normally after being shown to have either a balanced chromosomal translocation or (in two cases) an extra small chromosome of no clinical significance.

\section{Discussion}

Although there may come a time in the future when the majority of prospective parents request prenatal screening in every pregnancy, it would seem desirable and practical in the meantime to restrict this procedure to pregnancies in which the risk of fetal abnormality is substantially greater than the population risk. The arguments for selective screening are first that the skilled personnel and equipment which would be required for universal screening are not available and the cost of providing them would be prohibitive; secondly, the risks associated with early amniocentesis, at present small, would undoubtedly increase if it became a routine part of antenatal care; thirdly, the concept of selective termination of abnormal pregnancies is not universally accepted and at present insufficient is known about the emotional and psychological consequences.

The present series shows that the most important indication for fetal chromosome analysis, at least in numerical terms, is the risk of Down's syndrome in older mothers. In a recent retrospective Swedish study (Lindsjö, 1974) the risk of giving birth to children with trisomic Down's syndrome in mothers aged 35-39 years and 40-45 years was estimated to be $0.4 \%$ and $1.5 \%$ respectively. Although these rates are higher than those found in similar studies in the past, they represent less than $30 \%$ of the rates found prospectively in our study. Although our series is comparatively small, it appears to be consistent with the findings in other European centres (Galjaard, personal communication). The discrepancy between the retrospective and prospective findings is unlikely to be due entirely to the loss of trisomy 21 pregnancies between the time of amniocentesis and delivery, although it is conceivable that some of those detected by prenatal diagnosis would have been stillborn and unrecognized. A more likely explanation is that the frequency of Down's syndrome at birth has been consistently underestimated.

The conclusion that Down's syndrome is much more common than suspected in women over the age of 35 years has important implications for the provision of prenatal screening. If one in 20 of pregnant women aged 40 years and over are at risk of bearing a child with Down's syndrome, the argument for making prenatal diagnosis available to them is overwhelming. According to Lindsjö (1974), this group represents only $1.6 \%$ of all pregnancies so that the provision of the necessary facilities would not be insuperable. In the west of Scotland, with a population of just under 3 million, this would mean a maximum of 600 amniocenteses per annum. The number could be reduced further by a health education programme aimed to advise young women to complete their families by the age of 35 years, and to advise older women that prenatal screening is available.

Complete screening of the 40-years and older age group would reduce the birth incidence of Down's syndrome by about $21 \%$. Screening the much larger group of mothers aged between 35 and 40 years (6\% of all pregnancies) would detect $17 \%$ of Down's syndrome births. Evidence is presented elsewhere that economic benefits would balance costs if all mothers 35 years of age and over were included in a screening programme (Hagard and Carter, 1976). A lack of trained personnel and laboratory facilities means that it is impractical at present to attempt this on a wide scale, but this undoubtedly should be the aim for the future.

In younger mothers who have already had a child with trisomic Down's syndrome the empiric risk of recurrence estimated from retrospective studies is generally estimated to be about $1 \%$, and this is confirmed in the present prospective study. Many mothers have extreme anxiety about this compara- 
tively small risk and regard prenatal chromosome analysis as offering them the only chance to have a normal family. For this reason alone, it is important that prenatal screening should be made available to this group.

In the remaining categories of patients judged to be at risk of a fetal chromosome aberration the numbers involved are small although the risks tend to be higher. In theory the risk of a chromosomally unbalanced conception when the parent has a balanced chromosomal translocation is about $50 \%$, but in practice we find that prenatal testing at 16 weeks' gestation reveals that the risk is only about $5 \%$. One explanation is that the majority of abnormal conceptions are lost by spontaneous abortion before the time of amniocentesis. We have noted this occurrence in several of our patients booked for amniocentesis.

In cases of $\mathrm{X}$-linked recessive disease for which there is no specific biochemical test, selective termination on the basis of fetal sex may be the only alternative to risking a further affected child. For every affected male fetus aborted, on average one normal male fetus will be sacrificed. It is essential that research should be directed at establishing specific diagnostic tests for these disorders. We have been fortunate in our series in that of the five pregnancies at risk of Duchenne muscular dystrophy by chance four were female and could be saved. As early diagnosis of fetal sex is so important in these cases, we have felt it in order to advise the patient on the basis of sex chromatin examination of direct preparations of amniotic fluid cells if the fetus is female. Confirmation by fetal chromosome analysis is awaited before recommending termination of a male fetus. Although several centres regard fetal sex chromatin analysis as unreliable, we feel justified in using it by our successful experience with the Millipore filter technique (Ferguson-Smith et al, 1971). This is undoubtedly a major improvement in the technology of fetal nuclear sexing, as it provides fixation of amniotic cell nuclei much superior to that obtained in airdried smears. In all cases submitted for fetal sexing it is important that chromosome preparations from both parents should be examined for fluorescent polymorphisms and for $\mathrm{Y}$ chromosome variation so that Y-chromatin counts can be interpreted accurately.

Undoubtedly the major hazard in fetal chromosome analysis is the hazard of growing maternal cells instead of fetal cells. The growth of maternal cells tends to be associated with a difficult amniocentesis, attempted at too early a gestation, and a scanty blood-stained sample. The maternal cells appear to be exclusively fibroblast in type and it is likely that they are included in the sample either during inser- tion of the needle or during withdrawal when negative pressure is applied to the syringe. It is therefore essential to use a stilette with the needle and to discard the first and last few drops of amniotic fluid withdrawn into the needle. During culture it is unlikely that all primary cultures will be contaminated with maternal cells, and so an incorrect diagnosis is avoided if several cultures are analysed. The insitu method of analysis described here permits cytogenetic analysis of each clone separately which is a further safeguard. If the diagnosis has to be derived from one culture and if the cells have a female karyotype and are exclusively fibroblast in type, the mitoses should be examined for paternal chromosomal polymorphisms. If this fails to resolve the difficulty of distinguishing between fetal and maternal cells, the amniocentesis should be repeated.

A chromosome aberration arising by somatic mutation in amniotic cell cultures is a possible source of incorrect diagnosis. This is less likely to lead to difficulty if primary in situ type culture systems are used rather than systems in which chromosome preparations are made after typsinigation. In general, chromosome aberrations which are found in only one clone in one primary culture are likely to have arisen in vitro and should be disregarded.

Human cytogenetics is a highly specialized discipline and it seems clear to us that prenatal screening for fetal chromosome aberrations is the function of specialized laboratories with expertise in human cytogenetics and in cell culture technology. ldeally, such laboratories can be developed in departments which are already providing a cytogenetic diagnostic service on a regional basis. It is probably not practical to develop a prenatal diagnosis service as part of the routine work of a busy hospital pathological department. We are convinced that accurate genetic counselling is central to the provision of an effective prenatal screening programme, and that the most efficient results are obtained when the medical geneticist and the obstetrician work in close collaboration. The importance of ultrasound in ensuring safe and successful amniocentesis cannot be overemphasized.

\section{Summary}

Seven years' experience in prenatal screening for fetal chromosome aberrations in the west of Scotland is reviewed. Fetal chromosome analysis was undertaken in 716 pregnancies, $49 \%$ of which were judged to be at substantial risk of a fetal chromosome aberration. A chromosome aberration was found in 26 pregnancies, 14 of which were sufficiently severe to justify termination: the latter included nine cases of trisomic Down's syndrome, two cases of transloca- 
tion, two cases of XXY Klinefelter's syndrome and one case of the triple- $X$ syndrome. Ten pregnancies with balanced fetal chromosomal translocations and two with extra, small metacentric chromosomes of no clinical significance continued normally in pregnancy. Prenatal diagnosis permitted many mothers at risk the opportunity of having a family which otherwise they would not have attempted, and saved a number of pregnancies which would have been terminated solely on the risk, rather than the diagnosis, of fetal abnormality. An unexpectedly high frequency $(6.6 \%)$ of severe fetal chromosome aberrations was found in pregnancies of mothers aged 40 years and over. In the maternal age groups 35-39 years the frequency was $1.4 \%$. It is concluded that specialized cytogenetic facilities are urgently required to allow older mothers the option of prenatal screening. This is also required for younger mothers who have previously had a child with Down's syndrome, and for families at risk of chromosomal translocation and X-linked disease. Prenatal screening is best provided on a regional basis by a department of medical genetics experienced in genetic counselling, human cytogenetics, and cell culture techniques, working in close collaboration with obstetrical colleagues and the ultrasound department.

We are grateful to the National Fund for Research into Crippling Diseases for a generous grant to- wards prenatal screening in the west of Scotland, to Dr Hugh P. Robinson, Ultrasound Department, Queen Mother's Hospital, who performed a large proportion of the amniocenteses, to our numerous colleagues who referred patients, to Mrs Ann Theriault for her skilled assistance with amniotic cell cultures, and to members of the Medical Genetics Department, including Mrs Jill Gibson, Mrs Janet Jones, Mrs Margaret Al-Badran and Dr Elizabeth Boyd, for their help in various aspects of the work.

\section{References}

Ferguson-Smith, M. A. (1974). Prenatal diagnosis. The Practitioner, 213, 1277, 655-666.

Ferguson-Smith, M. E., Ferguson-Smith, M. A., Nevin, N. C., and Stone, M. (1971). Chromosome analysis before birth and its value in genetic counselling Brit. med.J., 4, 69-74.

Hagard, S., and Carter, F. A. (1976). Preventing the birth of infants with Down's syndrome: a cost-benefit analysis. Brit. med. J., 1, 753-756.

Harris, H. (1974). Prenatal Diagnosis and Selective Abortion. Nuffield Provincial Hospitals Trust, London.

Lindsjö, A. (1974). Down's syndrome in Sweden. Acta paediat. scand., 63, 571-576.

Milunsky, A. (1973). The Prenatal Diagnosis of Hereditary Disorders. Thomas, Springfield, Illinois.

Seabright, M. (1973). Improvement of trypsin method for banding chromosomes. (Letter). Lancet. 1, 1249-1250.

Sumner, A. T., Evans, H. J., and Buckland, R. A. (1971). New technique for distinguishing between human chromosomes. Nature [new Biol.], 232, 31-32. 